\title{
Indagine identitaria del personaggio seriale Verso una semiotica identitaria nella serialità
}

\author{
Nicolò Villani \\ Dipartimento delle Arti, Università di Bologna \\ nv.nico.villani@gmail.com \\ $<$ https://unibo.academia.edu/NicolòVillani>
}

\begin{abstract}
The contemporary media landscape is more and more pervaded by serial narrations called character-based. This tendency can be traced back to the dawn of the modern seriality, finding in characters as Sherlock Holmes the main cornerstones of popular serial narration. In all of this, a problem remains open: how are these characters able to keep their own identity through time and through the media? The topic of this paper is to try to answer the question placing the name of the character at the center of the problem from which to start the investigation of a concentric stratification of signification, by irradiation of a semic gradually more marginal up to the boundaries of the narrative context.
\end{abstract}

\section{Keywords}

Character; Identity; Sememe; Lexeme; Transmedia

\section{Sommario/Content}

1. Introduzione

2. Decostruire il personaggio

3. Un volto ai personaggi

4. Identità segrete

5. Ai confini del personaggio

6. Conclusioni

Bibliografia 


\section{Ocula}

Vol 21, No 22 (April 2020) • DOI: 10.12977/ocula2020-17

Nicolò Villani | Indagine identitaria del personaggio seriale. Verso una semiotica identitaria nella serialità

\section{Introduzione}

La proposta contenuta in questo saggio si situa all'interno del vasto panorama di studi inerente quel complesso e stratificato oggetto che è il personaggio, presentando una lettura del problema identitario che ne caratterizza la sua esistenza in un contesto sempre più transmediale; il personaggio in questione è quello seriale, che abita universi narrativi che non si limitano a un solo testo, ma che proseguono la propria esistenza in maniera continuata, attraversando il tempo e i media, coprendo diverse generazioni e sopravvivendo addirittura ai propri autori (cfr. Innocenti e Pescatore 2008). In un panorama di continua espansione e riscrittura, il personaggio configura strategie che gli permettano di resistere ai cambi di tempo, di contesto e di medium.

Il dibattito in merito, in ambito semiotico, ha molto spesso affiancato il problema a caratteri di ordine narrativo (cfr. Codeluppi 2015), andando a cercare l'efficacia della persistenza identitaria del personaggio inteso come transmediale (cfr. Jenkins 2010) all'interno del funzionamento delle narrazioni che lo vedono protagonista; ${ }^{1}$ il problema è stato inoltre affrontato cercando di mantenere costante lo specifico del medium, concentrandosi molto spesso su quei personaggi che trovano la loro fortuna nel contesto audiovisivo, parlando quindi del personaggio nel film (cfr. Comand 2012) o del cinema come dispositivo di traduzione di elementi testuali da un medium all'altro (cfr. Dusi 2003; 2015).

Questi approcci hanno il grosso pregio di tracciare delle descrizioni specifiche del funzionamento della cosiddetta "traduzione intersemiotica" (cfr. Pozzato 2014: 120 sgg.), dandone delle visioni tanto puntuali quanto ampie che comprendono la trasposizione dell'identità del personaggio nel più vasto orizzonte della ricostruzione del suo intorno narrativo, quale "collante contestuale" che ne sottoscriva la coerenza (cfr. Dusi 2003: 52 sgg.).

In tutto ciò, questo saggio vuole porsi su un piano diverso, che in qualche modo si inserisca nelle pieghe delle trattazioni sopra brevemente elencate, andando a intersecare la proposta di "eroe sociosemiotico" avanzata da Marrone (2003) che prosegue la strada avviata dai lavori di Eco sull'argomento ([1964] 2013, [1976] 2005): il personaggio può infatti trascendere la sua funzione puramente narrativa, consolidandosi come icona sociale, politica e, più ampiamente, culturale. In quest'ottica, l'identità del personaggio che qui si vuole indagare è quella che rimane costante pur trovandosi allontanata dal suo contesto narrativo di riferimento; come possiamo, ad esempio, affrontare la presenza dell'Uomo Ragno nelle pubblicità della Tim o spiegare la scena di Once Upon a Time in... Hollywood (C'era una volta a... Hollywood, Tarantino, 2019) in cui Cliff Booth schernisce Bruce Lee chiamandolo Kato? ${ }^{2}$ Allo stesso modo, come ricostruire i fondamenti su cui si basano fenomeni sociali

1 Nella trattazione si farà spesso riferimento a personaggi trattati all'interno dei saggi del volume di Codeluppi, ma, come si vedrà, l'approccio verterà su diversi elementi.

2 Il riferimento è al personaggio che Lee interpretò ai suoi esordi nella serie televisiva per famiglie Green Hornet (ABC, 1966-1967). 


\section{Ocula ${ }^{22}$}

Vol 21, No 22 (April 2020) • DOI: 10.12977/ocula2020-17

Nicolò Villani | Indagine identitaria del personaggio seriale. Verso una semiotica identitaria nella serialità

quali il cosplay o le comunità di fan che trascendono le componenti testuali che circondano i personaggi (cfr. Bisoni 2018 e Tralli 2018)?

La risposta a questo tipo di domande, che ipotizzano il personaggio in quanto oggetto significativo di per sé, ${ }^{3}$ a prescindere dal contesto narrativo, sarà condotta partendo da una suggestione barthesiana (cfr. Barthes [1970] 1973, tr. it.), dove il celebre autore, attraverso l'analisi di Sarrasine di Balzac, afferma che «quando semi identici attraversano a più riprese lo stesso nome e sembrano fissarvisi, nasce un personaggio», generando un prodotto combinatorio in cui il nome proprio funziona da campo magnetico degli elementi semici (ivi: 65). Il personaggio appare così una sorta di composto di elementi che ne hanno in qualche modo incrociato il nome, come attirati da una forza centripeta; per quanto prodotto ordinato, nella visione di Barthes, il personaggio/nome proprio appare troppo instabile per permettersi una sopravvivenza certa e continua. Infatti, l'aver considerato la presenza di componenti semiche all'interno del personaggio permette di legarlo alla definizione di unità lessemica che si dà del personaggio (cfr. Marsciani e Zinna 1991: 73), la visione di un accidentale attrattore di senso non ne permette la definizione come enunciato, originato da un'istanza e reso dunque discorso autonomo oltre che funzione. Il nome del personaggio va invece considerato come punto d'origine del corpo della significazione, cioè come «principio della donazione di senso» (Marsciani 2012: 148):

Il corpo sarebbe [...] una topologia che rende disponibili i posti che gli elementi in gioco vanno ad occupare, il corpo sarebbe quell'ordine o dimensione in cui le relazioni "prendono valore", in un processo di trasferimenti e trasformazioni che partono dal corpo e tornano al corpo, che cioè si danno come sue emergenze, come sue riformulazioni o metamorfosi, all'interno di un generale processo di organizzazione della materia. (ivi: 133)

Il nome/corpo, quindi, come nucleo puntiforme da cui viene irradiata significazione dove a riempire questa topologia stanno le componenti semiche, che sono gli elementi in grado di ricondurre al senso un enunciato. Il nome proprio resta perciò come punto di partenza (e non di semplice congiunzione) fondamentale (cfr. Casetti e Di Chio 1990: 167), originando la messa in discorso del personaggio stesso, in un intorno di componenti semiche che rendono l'attore da lessema, un semema pienamente significante e riconoscibile.

Quello di cui ci si vuole occupare qui è, quindi, individuare e classificare le componenti semiche, posizionandole nel giusto rapporto con la natura irradiante del personaggio, valutandone le funzioni e le capacità di attribuire al personaggio stesso il proprio senso. Per farlo si andranno ad analizzare esempi tra i più rappresentativi della natura seriale che un personaggio può vivere cercando di estrapolare da questi casi particolari dei criteri che abbiano un

3 In questo saggio si parlerà di "personaggio" piuttosto che di "eroe" proprio per sottolineare questo distacco dal contesto narrativo. Le teorie qui descritte possono essere idealmente applicate a qualsiasi tipo di personaggio, a prescindere dalla sua importanza. 


\section{Ocula}

Vol 21, No 22 (April 2020) • DOI: 10.12977/ocula2020-17

Nicolò Villani | Indagine identitaria del personaggio seriale. Verso una semiotica identitaria nella serialità

valore generale; ogni caso preso in considerazione possiede le seguenti caratteristiche:

- vive una natura plurimediale, avendo intersecato ciascuno dei tre media seriali principali qui presi in considerazione (letteratura, fumetto, audiovisivo);

- è definibile come prodotto del settore character-based entertainment;

- porta con sé un nutrito fandom, variabilmente attivo, che dà origine a pratiche definite grassroots (cfr. Jenkins [2006] 2014, tr. it.).

\section{Decostruire il personaggio}

\subsection{Sherlock Holmes: il personaggio seriale moderno}

Nato nel 1887 dalla penna di Sir A. Conan Doyle, Sherlock Holmes si può ritenere uno dei massimi esempi di personaggio seriale moderno, poiché già dalla sua prima forma espressiva è stato oggetto di continua attenzione di lettori e critica, fino a creare quello che oggi sarebbe definito un autentico fandom (cfr. Cucchetti 2014: 73-75). Già ai suoi albori, il detective si vede protagonista di più forme espressive, come il teatro e il cinema. Nel corso dei decenni, sono stati tantissimi i volti mediali di Holmes, quante le sue riscritture, insieme a innumerevoli rappresentazioni. Dove sta, dunque, nella varietà dei prodotti, quell'elemento che permette di identificare proprio Sherlock Holmes?

Roberta Pearson definisce il personaggio come un

"network of rappresentations" comprised of a "transmedia structure" of all the books, magazines, advertising, films, radio and television programs, YouTube videos, comic books, games, toys, fan fictions, and so forth that have accumulated since 1887. Pearson (2016: 79)

Per individuare gli elementi costanti e via via introdotti nella "struttura transmediale" che è il personaggio di Holmes, si può tentare una sua decostruzione attraverso le proprie fasi salienti, individuando quegli attributi che vanno a caratterizzarlo come riconoscibile.

La prima descrizione ci viene data dallo stesso Doyle nel primo romanzo:

La statura di Holmes superava il metro e ottanta ed era tanto magro che sembrava più alto. Aveva gli occhi acuti e penetranti [...]; il naso, affilato e un po' adunco, conferiva al viso un'espressione vigilante e decisa. Anche il mento, quadrato e pronunciato, denotava in lui una salda volontà. (Doyle [1887] 2010, tr. it)

Leggendo il resto del Canone si viene a conoscenza della predilezione di Holmes per il fumo e per l'uso della lente d'ingrandimento, nonché di alcuni atteggiamenti ricorrenti.

Per le successive fasi, pare opportuno limitarsi a quegli interpreti che hanno rappresentato «the quintessential Holmes of their generations»: William Gillette, Basil Rathbone, Jeremy Brett e Benedict Cumberbatch (cfr. Pearson 2016: 77, 82). 


\section{Ocula $^{22}$}

Vol 21, No 22 (April 2020) • DOI: 10.12977/ocula2020-17

Nicolò Villani | Indagine identitaria del personaggio seriale. Verso una semiotica identitaria nella serialità

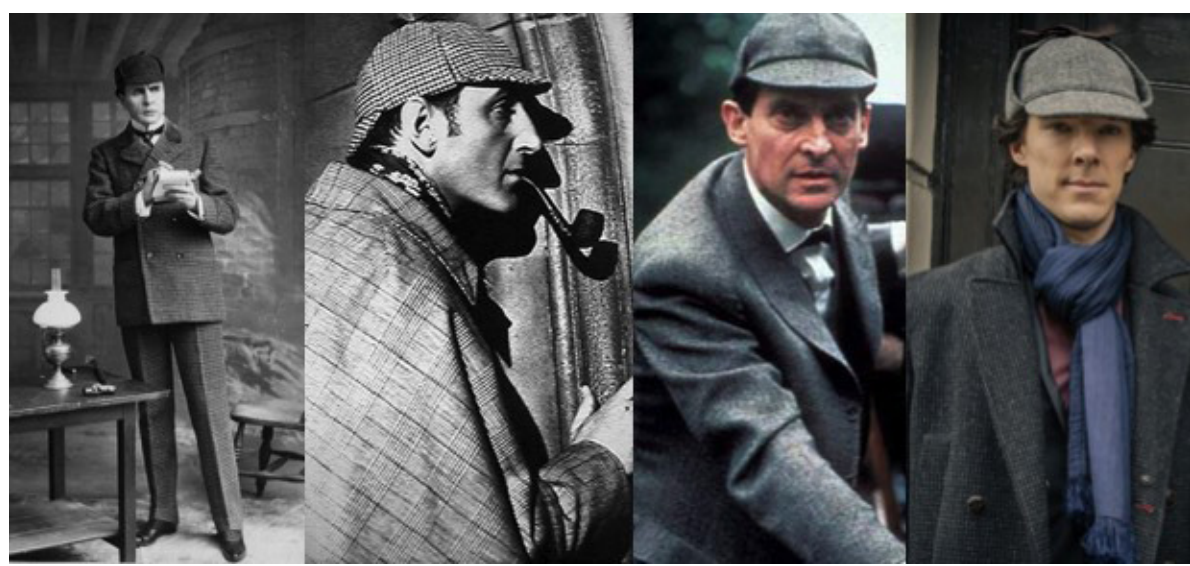

Figura 1. Da sinistra, Gillette, Rathbone, Brett e Cumberbatch.

Proprio con Gillette possiamo individuare gli elementi tipici del vestiario, come il cappello e il cappotto; lo stesso Gillette ha introdotto, nello spettacolo teatrale Sherlock Holmes (1899), la frase «elementare, Watson», assente nel Canone; con ben quattordici pellicole dal 1939, Rathbone conferma la presenza degli elementi introdotti da Gillette, dando centralità alla pipa.

La serie tv The Adventures of Sherlock Holmes (Le Avventure di Sherlock Holmes, Granada, 1984-1994), con protagonista Brett, riporta il personaggio a una maggior vicinanza al Canone, pur mantenendo il cappello e il cappotto. In fine, con Cumberbatch e la serie tv Sherlock (Id., BBC, 2010-in corso), il detective viene trasportato nel contemporaneo, mantenendo i riferimenti alle versioni precedenti; l'Holmes di Cumberbatch è più emotivo, nonché immerso in una Londra all'avanguardia.

\subsection{Intersemiosi e forme semiche}

I casi sopra rapidamente elencati sono esempi di come sia evoluta la "struttura transmediale" di Sherlock Holmes in quanto personaggio, attraverso quelle riscritture definibili come traduzioni intersemiotiche, passaggi di medium che costringono a selezionare quali elementi tenere dai media precedenti e quali modificare (cfr. Pozzato 2014: 120). Gli elementi minimi sopra descritti si possono definire come componenti semiche delle varie traduzioni intersemiotiche di Sherlock Holmes. Partendo da ciò, si può osservare come lo stesso personaggio viva una duplice natura: è lessema quando deve essere ancora "riempito" con i semi opportuni e semema una volta che la sua significazione è completa, coerente e riconoscibile. La semiotica propone una duplice divisione delle componenti semiche: semi nucleari e semi contestuali; i primi si riferiscono alle qualità proprie del lessema/semema, mentre i secondi lo collocano in un intorno di senso per, appunto, contestualizzarlo (cfr. Marsciani e Zinna 1991: 40).

Si possono ora collocare gli elementi individuati nel paragrafo precedente dando una classificazione alle varie componenti semiche. Partendo dalle pre- 


\section{Ocula}

Vol 21, No 22 (April 2020) • DOI: 10.12977/ocula2020-17

Nicolò Villani | Indagine identitaria del personaggio seriale. Verso una semiotica identitaria nella serialità

messe poste dai romanzi si è in grado di porre il personaggio nel suo contesto: saranno quindi semi contestuali Londra, l'età Vittoriana, l'ambito investigativo, ecc. Sempre dai romanzi, veniamo a conoscenza di una sommaria descrizione fisica del personaggio con espressioni come «occhi acuti e penetranti» che aiutano a definire l'aspetto fisico di Holmes e che si potranno chiamare semi nucleari fisici. Sempre a livello visivo, gli elementi di vestiario rientrano in una loro classe di semi nucleari, definibili plastici. Il cappello può avere inoltre una seconda funzione: si tratta di un elemento talmente legato al personaggio da poterne fare le veci con la sua sola presenza; lo stesso vale per la pipa o il dottor Watson. Questi elementi, al confine tra i semi nucleari plastici e i semi contestuali, sono i semi nucleari circostanti. Sono poi semi nucleari caratterizzanti tutti quelli che delineano nel personaggio atteggiamenti e azioni tanto da renderli prevedibili in "situazioni tipo", come la capacità deduttiva, il rapporto con Watson, l'opinione sulle capacità della polizia, l'amore per il violino e il distacco da ogni sentimento romantico.

Questo piccolo riassunto delle componenti semiche appare sufficiente per delineare un "campo di riconoscibilità" di Sherlock Holmes minimo da mettere alla prova attraverso la prova di commutazione.

\subsection{Commutare Sherlock Holmes}

Come si è detto, Holmes ha trovato continue evoluzioni e ridefinizioni, fino, in alcuni casi, a distaccarsi dall'originale. Ma dunque, quali componenti semiche fanno del nome "Sherlock Holmes" proprio quel semema? La risposta non può che essere: dipende.

Attraverso l'efficace mezzo della prova di commutazione, si può andare a verificare, variando una delle componenti semiche individuate con una corrispondente sull'asse paradigmatico, quanto questa variazione risulti significativa (cfr. Marsciani e Zinna 1991: 23 sg.). Un esempio di commutazione espressiva è quella di sostituire i semi nucleari fisici con il volto e il corpo di Basil Rathbone, nella trasposizione cinematografica: è evidente che l'operazione non risulti significativa, in quanto la descrizione letteraria (abbastanza generica) si possa adattare al volto dell'attore; a rinforzare questo passaggio concorre la presenza di altri gruppi di semi nucleari, come i plastici e i circostanti. ${ }^{4}$

Un repentino cambio di contesto, a sua volta, non basta da solo a far perdere al personaggio la sua identità, come dimostra Sherlock, in cui Benedict Cumberbatch interpreta un Holmes inserito nella Londra contemporanea, mantenendo una notevole quantità degli altri gruppi semici nucleari.

Più complesso è il riconoscimento del personaggio quando ai semi nucleari fisici si sostituiscono quelli di un animale, come nel caso di Basil The Great Mouse Detective (Basil l'investigatopo, Clements e Musker, 1986): in questo caso Holmes è solo una componente intertestuale richiamata dall'uso di precisi semi nucleari appartenenti al detective (Dusi 2019: 109 sg.).

4 Per una trattazione più dettagliata e criticamente accurata della trasposizione cinematografica da medium letterario, si rimanda nuovamente a Dusi (2019). 


\section{Ocula}

Vol 21, No 22 (April 2020) • DOI: 10.12977/ocula2020-17

Nicolò Villani | Indagine identitaria del personaggio seriale. Verso una semiotica identitaria nella serialità

Diventa invece più rischioso dal punto di vista della significazione sacrificare un numero eccessivo di componenti semiche in più gruppi; ne è un esempio l'interpretazione cinematografica di Robert Downey Jr. (2009-2011), dove il personaggio è riconoscibile unicamente attraverso classemi e semi nucleari caratterizzanti.

Nei prossimi tre paragrafi si darà spazio a tre dei gruppi semici individuati: fisici, plastici e circostanti; si tralasceranno volutamente le dimensioni caratteriali e contestuali, più legate agli aspetti di teoria della sceneggiatura (cfr. Bandirali e Terrone 2009) e a questioni di stampo narrativo, già ampiamente considerate nei testi citati in introduzione.

\section{Un volto ai personaggi}

\subsection{Star Wars: una galassia di attori}

Apparso sugli schermi cinematografici nel 1977, l'universo narrativo di Star Wars è uno dei primi esempi di narrazione transmediale; già dopo l'uscita della prima pellicola si ebbe un'enorme produzione di gadgets e merchandising che ritraevano la folta mitologia ideata da George Lucas. Jenkins ([2006] 2014: 131-178, tr. it.) individua in Star Wars l'esempio di un prodotto di cui i fan possono appropriarsi per creare nuove narrazioni; ma perché un universo simile possa funzionare anche oltre alla narrazione ufficiale, è necessario che i suoi personaggi siano chiaramente riconoscibili (ivi: 148).

A differenza di Sherlock Holmes, i personaggi di Star Wars nascono per un medium di natura visiva, quindi ogni attore è scelto per quello che il personaggio rappresenterebbe in una sua eventuale figurazione "letteraria". Allo stesso tempo i volti degli attori diventano i volti dei gadget, quindi devono avere dei tratti unici e facilmente riproducibili. Il compito di Lucas al momento del casting è, quindi, trovare persone che esprimano una certa unicità fisica, sia nel volto che nei movimenti (e persino nella voce), per poterne facilmente commercializzare l'immagine. Come si vede nella figura 2, il passaggio dagli artworks preparatori al personaggio dimostra l'importanza che Lucas attribuisce alla fisicità dell'attore piuttosto che agli elementi di contorno; la stessa fisicità viene inoltre mantenuta al momento della realizzazione delle action figure e del resto del merchandising.

$\mathrm{Ma}$, in questo caso, quanto è inscindibile il legame tra attore e personaggio? La risposta a questa domanda va cercata analizzando quelli che nel paragrafo precedente abbiamo chiamato semi nucleari fisici.

\subsection{Il corpo come figura}

Considerando il personaggio come un punto dal quale vengono irradiate in aree concentriche le sue componenti semiche, divise come indicato nel precedente paragrafo, i semi nucleari più "aderenti" a esso sono i fisici, "nucleari" in quanto appartengono al personaggio a prescindere dal contesto e "fisici" poiché riguardano la sua descrizione fisica, somatica. Fanno parte di questo 


\section{Ocula $^{22}$}

Vol 21, No 22 (April 2020) • DOI: 10.12977/ocula2020-17

Nicolò Villani | Indagine identitaria del personaggio seriale. Verso una semiotica identitaria nella serialità

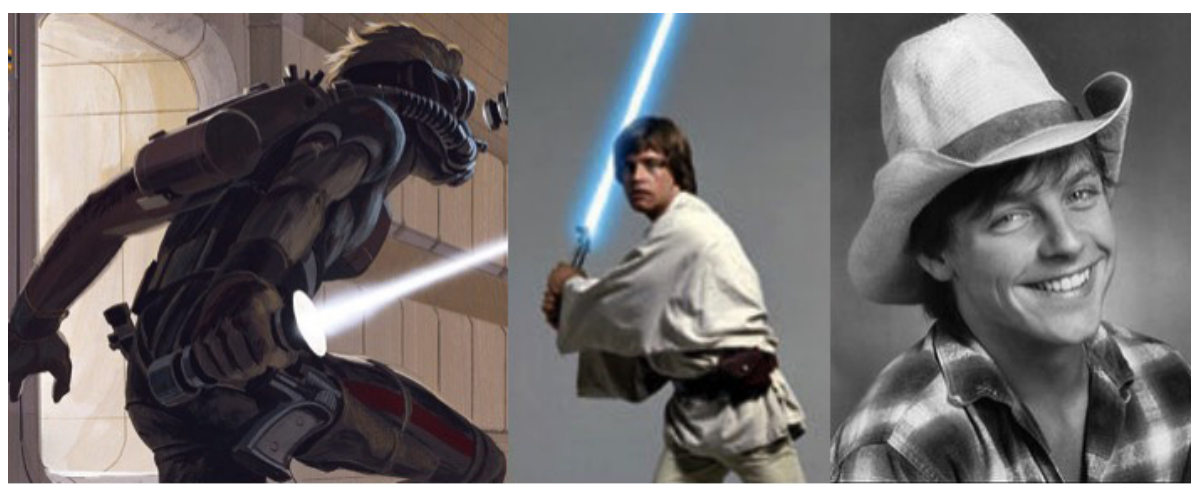

Figura 2. Da sinistra, artwork di Ralph McQuarrie, Luke Skywalker e Mark Hamill.
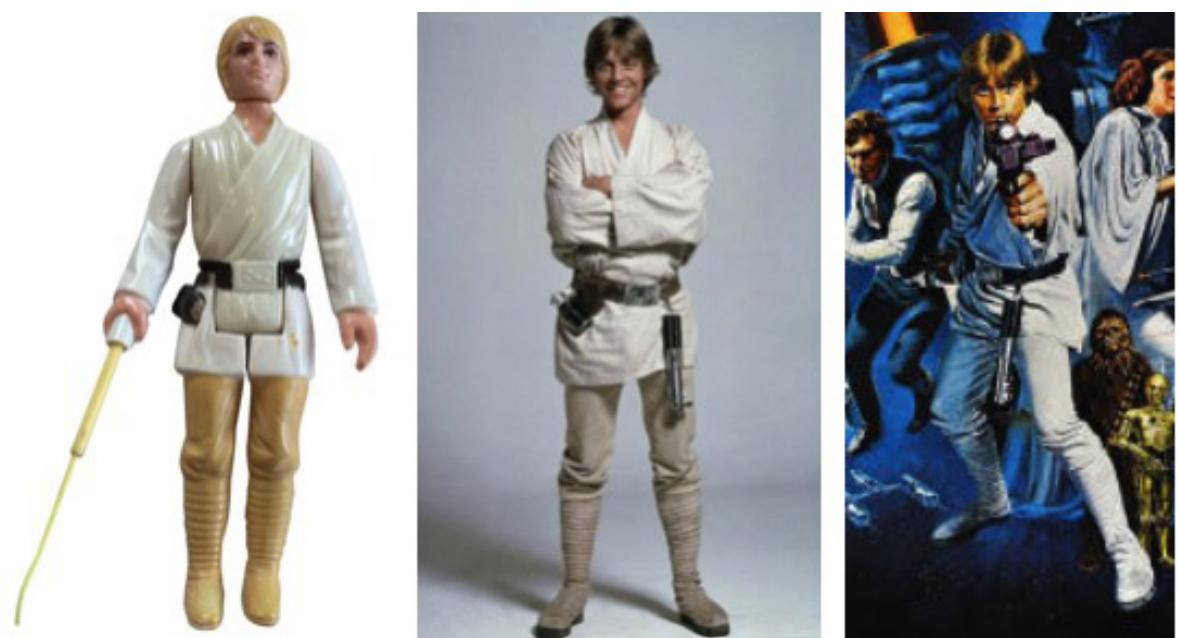

Figura 3. Da sinistra, action figure di Luke Skywalker, Luke Skywalker e illustrazione promozionale del personaggio.

gruppo i tratti del volto, l'altezza, la taglia, la postura, la voce, ecc. nelle più disparate combinazioni, sia che questi siano esplicitati o meno. A seconda del medium veicolante, i semi nucleari fisici possono avere maggiore o minore definizione, descrivibile attraverso i cosiddetti gradi d'iconizzazione, che dipendono fortemente dall'aderenza con la realtà delle figure rappresentate (cfr. Marsciani e Zinna 1991: 118). ${ }^{5}$ Un linguaggio letterario tenderà a dare una descrizione che resterà sempre e comunque aperta ad aggiunte e interpretazioni: come visto precedentemente per Sherlock Holmes, si può provare a descrivere Luke Skywalker in modo da comprendere caratteristiche fisiche e tematiche verificando il grado d'iconizzazione che ne risulta: «Un giovane biondo con gli

5 Va sottolineato come l'iconizzazione si deve intendere come un punto di arrivo che mette al riparo da considerare l'esistenza di linguaggi più iconici di altri (cfr. Marsciani e Zinna 1991: 118). 


\section{Ocula ${ }^{22}$}

Vol 21, No 22 (April 2020) • DOI: 10.12977/ocula2020-17

Nicolò Villani | Indagine identitaria del personaggio seriale. Verso una semiotica identitaria nella serialità
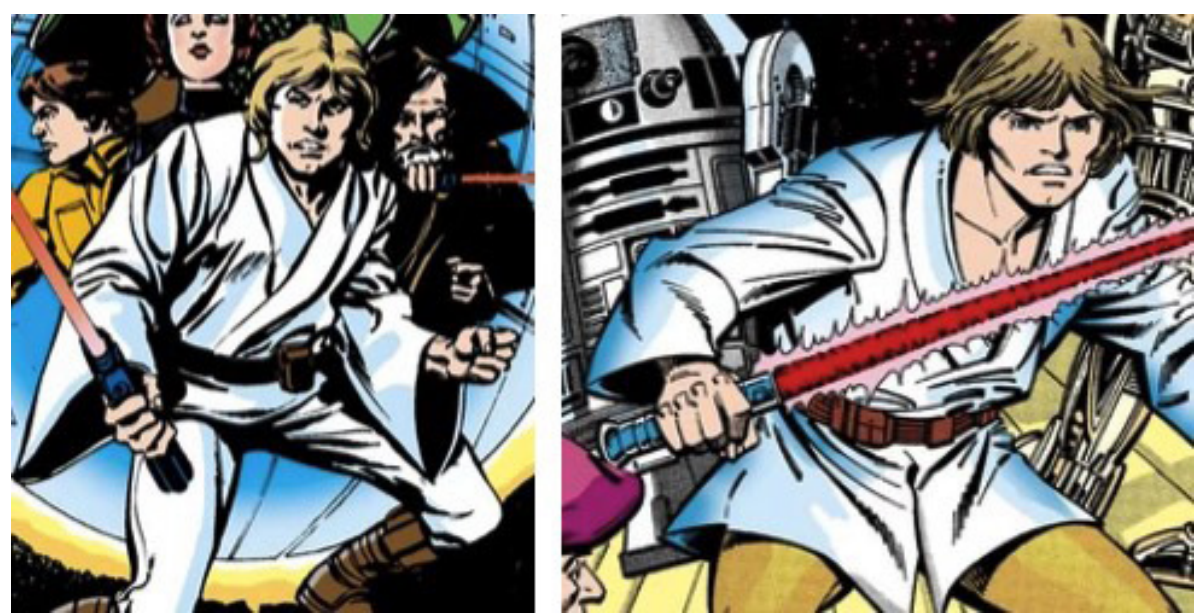

Figura 4. Da sinistra, Luke Skywalker disegnato da Howard Chaykin e da Carmine Infantino.

occhi azzurri, inesperto, con l'aria da bravo ragazzo, che trasmette intelligenza e integrità morale».

Poco altro si potrebbe aggiungere a questo insieme mai completo di dati sommari; una descrizione come quella proposta sopra sarebbe comunque sufficiente per permettere a un illustratore di realizzare un'immagine del personaggio.

Entrambe le illustrazioni in figura 4 rispettano la descrizione letteraria, ma i due personaggi appaiono differenti per molti dettagli. Ulteriori gradi d'iconizzazione si acquistano con l'animazione o con la realizzazione di action figure. Tutti questi prodotti hanno una loro scala d'iconizzazione, ma al vertice di ognuna non si raggiunge mai la completezza iconica raggiungibile dall'utilizzare un vero corpo umano con tutte le sue caratteristiche da donare al personaggio per farne quel modello da cui tutti gli altri media devono trarre necessariamente quanto possibile perché questi sia sempre sé stesso. Il personaggio diventerà quindi un sosia della persona che gli presta le caratteristiche fisiche.

\subsection{L'effetto sosia: anatomie in prestito}

Si può definire effetto sosia quel particolare caso in cui un personaggio acquisisce una gran quantità di semi nucleari fisici da una persona reale raggiungendo il grado massimo d'iconizzazione permesso dal medium. Si può dire che i personaggi che raggiungono questo grado d'iconizzazione "assomiglino moltissimo" a determinati attori; impersonare un personaggio che non possiede ancora un proprio volto definito significa, infatti, far sì che quell’identità priva di contenuti fisici si appropri di quelli dell'attore: non si dirà, ad esempio, che Han Sono è Harrison Ford, bensì che entrambi condividono i medesimi semi nucleari fisici; di conseguenza, se un personaggio condivide il volto con un attore che ha interpretato altri personaggi, egli sarà un sosia 


\section{Ocula $^{22}$}

Vol 21, No 22 (April 2020) • DOI: 10.12977/ocula2020-17

Nicolò Villani | Indagine identitaria del personaggio seriale. Verso una semiotica identitaria nella serialità

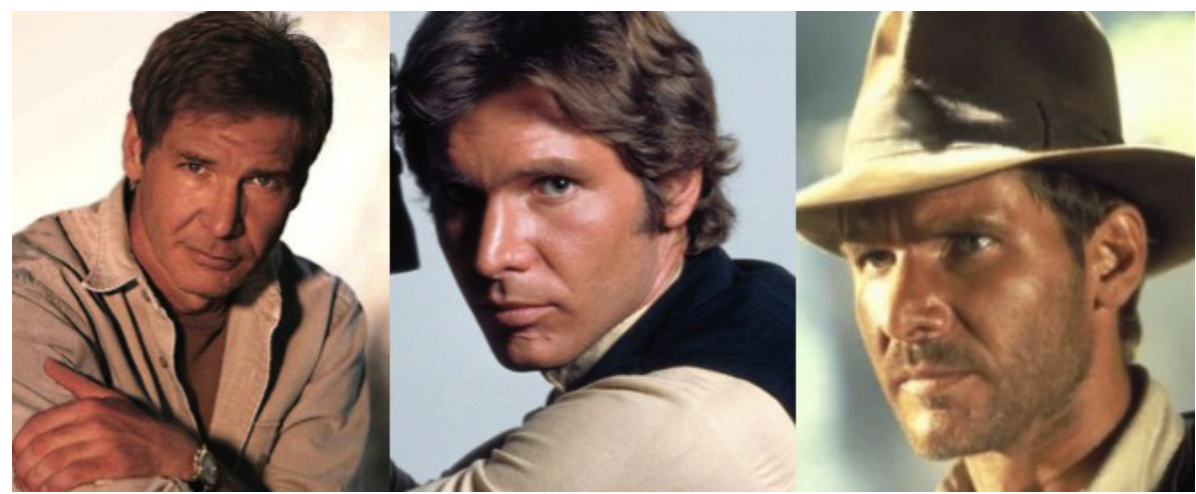

Figura 5. Da sinistra, Harrison Ford, Han Solo e Indiana Jones.

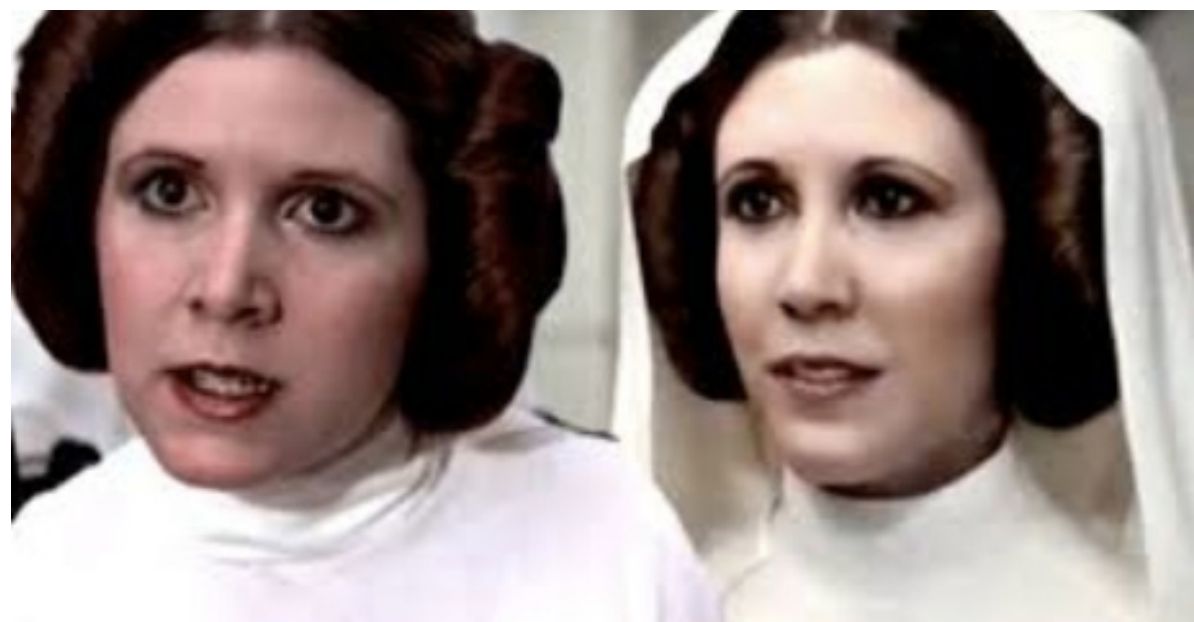

Figura 6. Da sinistra, la principessa Leia in Star Wars (Guerre Stellari, George Lucas, 1977) e in Rogue One.

anche di questi ultimi: si può facilmente notare come Harrison Ford sia sosia tanto di Han Solo quanto, ad esempio, di Indiana Jones. ${ }^{6}$

Lo stesso si applicherà, quindi, alle espansioni transmediali dei personaggi, che vedranno applicare adattamenti dei volti degli attori nei vari prodotti. Dalle parole di Carrie Fisher: «Non sei famoso finché non sei un contenitore di caramelle. [...] Non sono famosa io, è famosa la principessa Leia; io ho la sua faccia!» ${ }^{7}$

Vi sono poi casi in cui il personaggio non si limita ad attingere i suoi semi nucleari fisici da un'unica persona, bensì cercandoli in più individui, come nel caso di Darth Vader, la cui fisicità è resa dalla somma di caratteristiche di diversi attori.

6 Per approfondimenti sul personaggio si veda Bonvecchio (2011).

7 Da un'intervista contenuta in Empire of Dreams: The Story of the 'Star Wars' Trilogy (L'impero dei sogni: la storia della trilogia di Star Wars, Becker e Burns, 2004). 


\section{Ocula}

Vol 21, No 22 (April 2020) • DOI: 10.12977/ocula2020-17

Nicolò Villani | Indagine identitaria del personaggio seriale. Verso una semiotica identitaria nella serialità

Ma in definitiva, quanto è vincolante l'effetto sosia? Questo appare vincolante ogni qual volta si vada a rappresentare il personaggio in un'età molto vicina o successiva a quella in cui si solidifica l’identità; Harrison Ford deve per forza essere l'Han Solo più anziano e, allo stesso tempo, nessun'attrice può interpretare la principessa Leia nell'ultima scena di Rogue One: A Star Wars Story (Id., Edwards, 2016), costringendo all'uso della computer grafica.

$\mathrm{Al}$ contrario è possibile aggirare l'effetto sosia quando si rappresenta il personaggio in un periodo della sua vita molto precedente rispetto a quello della prima apparizione.

L'effetto sosia permette di solidificare l'identità visiva del personaggio, dandone un insuperabile grado d'iconicità, in modo che ne veicoli al meglio le componenti tematiche.

\section{Identità segrete}

\subsection{Uomini e superuomini}

Con i suoi oltre ottomila personaggi, la Marvel Comics è una delle più grandi aziende del settore del character-based entertainment; con l'inizio degli anni Sessanta, l'Universo Marvel inizia a popolarsi in maniera esponenziale di eroi sempre nuovi, con i più svariati superpoteri e super-problemi. Le tavole a fumetti diventano così teatro di coloratissime avventure, con protagonisti in calzamaglia, spesso dotati di identità civili da mantenere segrete e vite normalissime da affiancare a quelle straordinarie vissute con indosso la maschera.

Il medium fumettistico pone un problema a un universo narrativo popolato da tantissimi personaggi: il basso grado d'iconicità; questa caratteristica pone l'accento sulla difficoltà di distinguere i volti e di individuare i personaggi al momento dell'eventuale cambio di disegnatore; la caratteristica dei personaggi Marvel è, fin dall'inizio, quella di vivere in un universo comune, interagendo e incontrandosi, trovandosi quindi a comparire in svariate testate, spesso curate da disegnatori differenti, che segnano col loro tratto unico praticamente ogni personaggio dell'universo narrativo. Al contempo, un linguaggio come quello dei fumetti di supereroi si presta alla trasposizione nel suo medium narrativamente più vicino: il cinema (cfr. Barbieri 1991). Come attesta Claudio Bertieri (1979: 36-37), fin dal 1944, con il serial cinematografico dedicato a Capitan America, i supereroi hanno preso nuova vita sullo schermo. Questa tendenza, mai interrotta e più volte ricomparsa, ha trovato una forte volontà di affermazione con il Marvel Cinematic Universe, esempio di universo narrativo cinematografico in continuity nato per persistere. Ma il cinema pone problemi analoghi: come restano riconoscibili sullo schermo personaggi nati su carta e con un basso grado d'iconizzazione, cambiando spesso il volto di chi li interpreta?

La soluzione ci viene suggerita da Valentina Semprini (2006: 90): «Il costume serve [...] a identificare il personaggio, a distinguerlo dagli altri e, talvolta, a dare qualche informazione su di lui.» La costante che permette a un supereroe di distinguersi dagli altri, qualsiasi sia il medium, è il suo costume e le parti di cui è composto possono rientrare nella categoria dei semi nucleari plastici. 


\section{Ocula $^{22}$}

Vol 21, No 22 (April 2020) • DOI: 10.12977/ocula2020-17

Nicolò Villani | Indagine identitaria del personaggio seriale. Verso una semiotica identitaria nella serialità

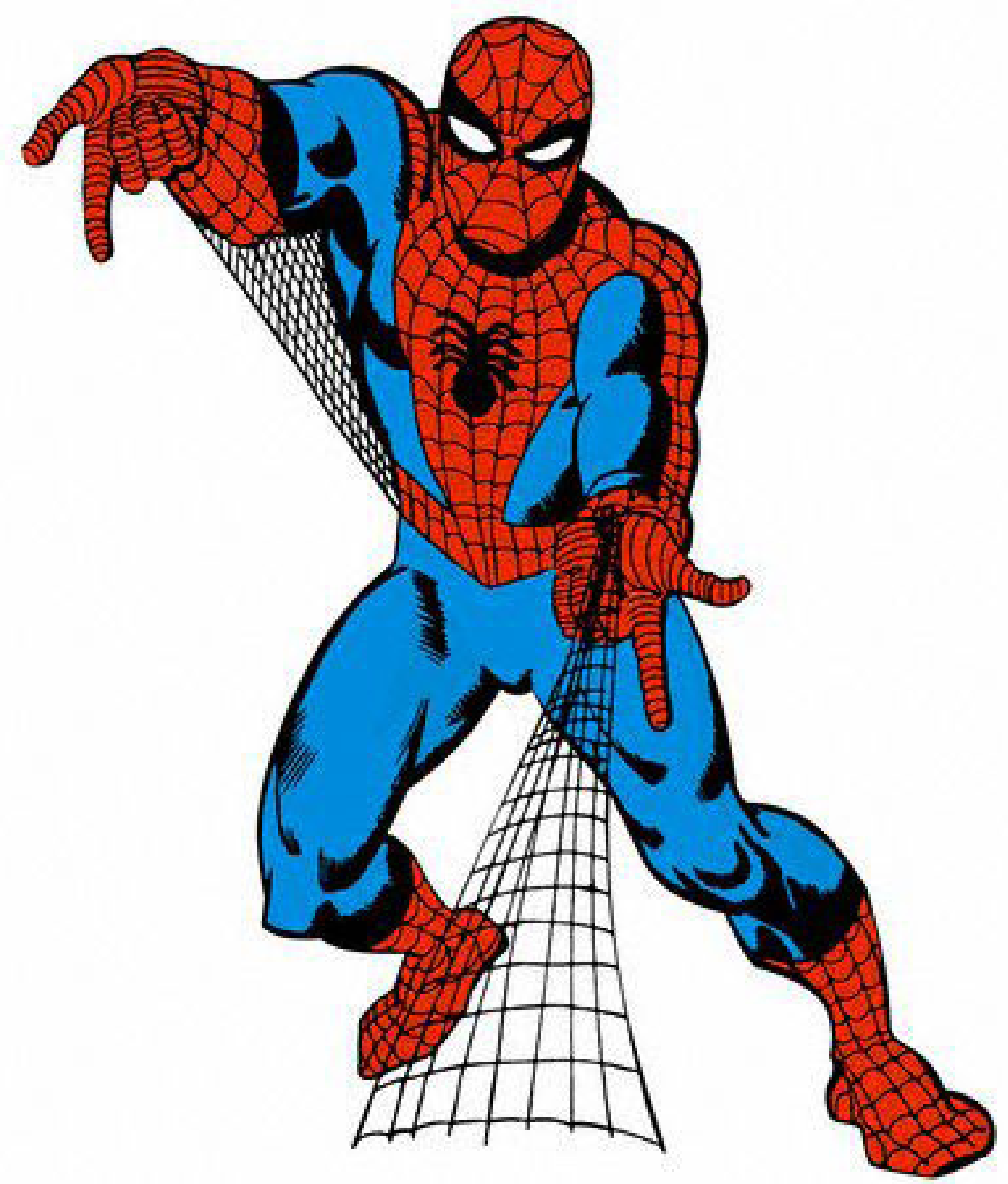

Figura 7. L’Uomo Ragno disegnato da Steve Ditko.

\subsection{La maschera oltre il volto}

Se i semi nucleari fisici rappresentavano lo strato più aderente all'ipotesi puntiforme del personaggio irradiante significazione, quelli immediatamente dopo sono i semi nucleari plastici, "avvolti" intorno a quelli fisici; si definiscono "plastici" poiché sono in grado di raggiungere elevati gradi di astrazione semantica e riguardano quei formanti visivi che prescindono le figure (Greimas 1984).

I costumi dei supereroi si prestano all'esemplificazione di questo gruppo di semi nucleari, vestendo in maniera aderente il corpo del personaggio; allo stesso tempo, la loro ricchezza grafica permette di suddividere le varie componenti semiche di ogni costume classificandole in una scala di densità semanti- 


\section{Ocula $^{22}$}

Vol 21, No 22 (April 2020) • DOI: 10.12977/ocula2020-17

Nicolò Villani | Indagine identitaria del personaggio seriale. Verso una semiotica identitaria nella serialità

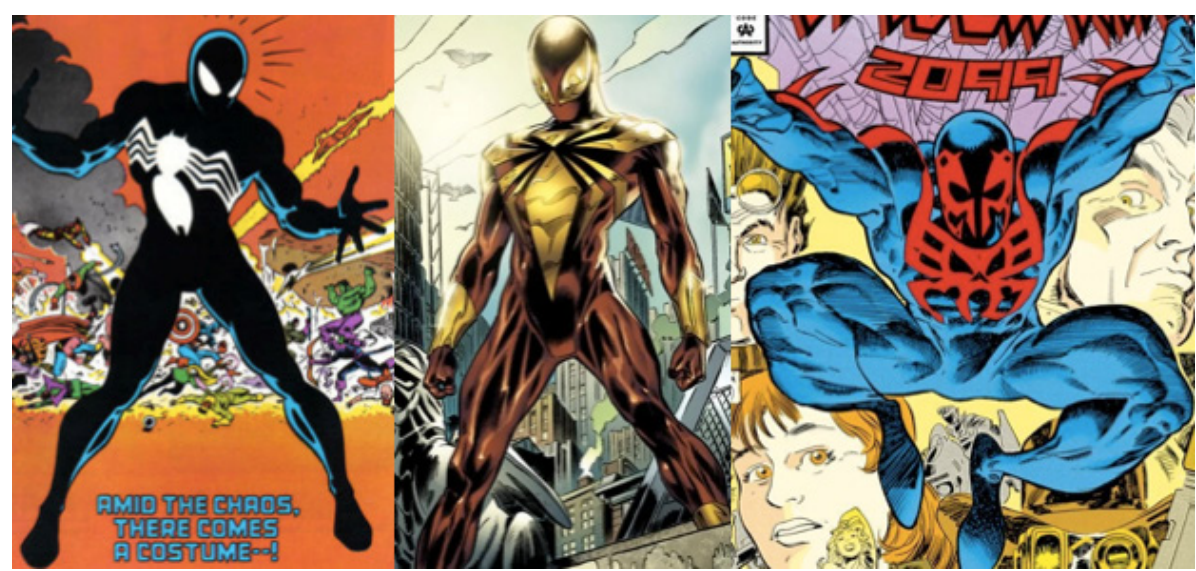

Figura 8. Tre famose varianti del costume dell’Uomo Ragno.

ca: i costumi dei supereroi altro non sono, infatti, che risultati di una semantica componenziale le cui parti hanno tra di loro un rapporto eterogeneo di densità (Pozzato 2014: 41-54).

Useremo come esempio uno dei costumi più famosi e duraturi della storia dei fumetti di supereroi, quello dell'Uomo Ragno.

La prima componente plastica con la più bassa densità semantica è quella cromatica: i contrasti tra rosso e blu permettono di distinguere varie parti del costume. Dal punto di vista eidetico, troviamo parti variabilmente dense: i contorni che separano le zone cromatiche delimitando aree all'interno dell'anatomia; gli occhi della maschera ricordano un'espressione sinuosa e aggressiva; inoltre, ad un grado di densità più elevato, si trovano le ragnatele che ricoprono le zone rosse del costume, sottolineando la natura "ragnesca". Ultimo elemento, ad elevatissima densità semantica, è il ragno al centro del petto.

Servendosi nuovamente della prova di commutazione si andrà ora a verificare quali elementi risultano significativi in eventuali varianti del costume classico perché il personaggio resti riconoscibile.

Nel primo caso, in figura 8, scompaiono il contrasto cromatico e le ragnatele; si mantengono il ragno e le lenti della maschera. Nel secondo caso il contrasto cromatico viene sfruttato per rimandare a un altro supereroe, Iron Man, con un effetto intertestuale; qui i contorni vanno a delimitare poche aree e nuovamente si perdono le ragnatele, pur mantenendosi le lenti. Nel terzo caso, il contrasto cromatico resta; il ragno assume un aspetto inquietante, seppur riconoscibile; le ragnatele anche qui spariscono e l'effetto espressivo delle lenti viene reso dagli elementi interni della maschera.

Siamo ora in grado di fare un bilancio degli elementi fondamentali perché la semantica componenziale del costume permetta il riconoscimento: il contrasto cromatico, pressocché superfluo può essere sfruttato come rimando intertestuale; l'elemento eidetico che va a collegarsi con altri semi nucleari (in questo caso caratterizzanti) si mantiene assumendo la funzione di anticipazione di questi; infine, quando un elemento ha la stessa funzione di un altro, con una densità semantica inferiore, può essere trascurato senza che ne risenta 


\section{Ocula ${ }^{22}$}

Vol 21, No 22 (April 2020) • DOI: 10.12977/ocula2020-17

Nicolò Villani | Indagine identitaria del personaggio seriale. Verso una semiotica identitaria nella serialità

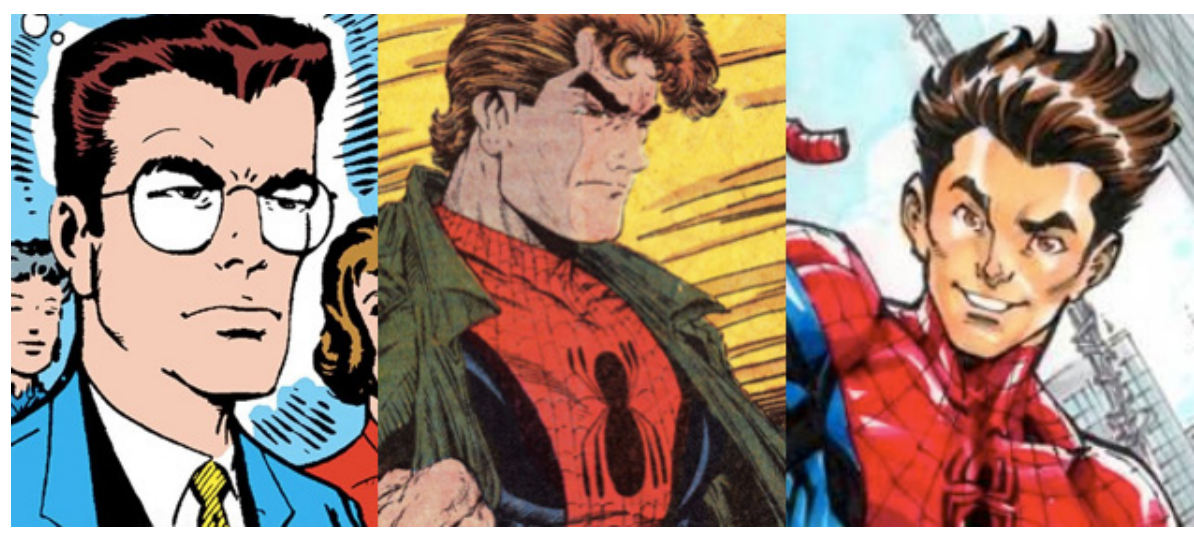

Figura 9. Da sinistra, Peter Parker disegnato da Steve Ditko, Todd McFarlane e Todd Nauck.

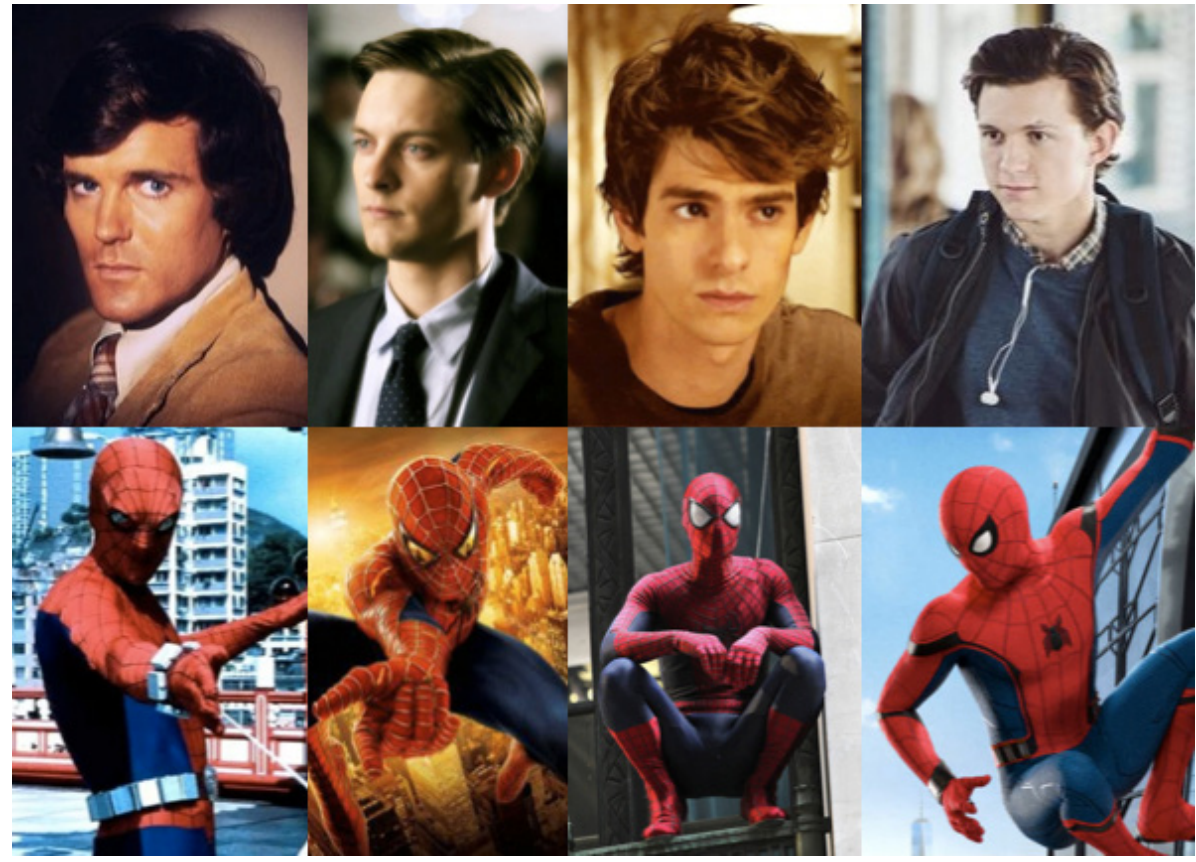

Figura 10. Da sinistra, Peter Parker interpretato da Nicholas Hammon, Tobey Maguire, Andrew Garfield e Tom Holland.

la significazione complessiva (è il caso delle ragnatele che veicolano lo stesso contenuto del simbolo del ragno). Resta ora, però, ancora da prendere in considerazione l'elemento dell'identità segreta.

\subsection{Identità avvolgenti}

Sotto la maschera del supereroe c'è molto spesso una persona comune, con un proprio volto e una propria identità, che il medium fumettistico fatica a rendere univoca dal punto di vista fisico. 


\section{Ocula $^{22}$}

Vol 21, No 22 (April 2020) • DOI: 10.12977/ocula2020-17

Nicolò Villani | Indagine identitaria del personaggio seriale. Verso una semiotica identitaria nella serialità

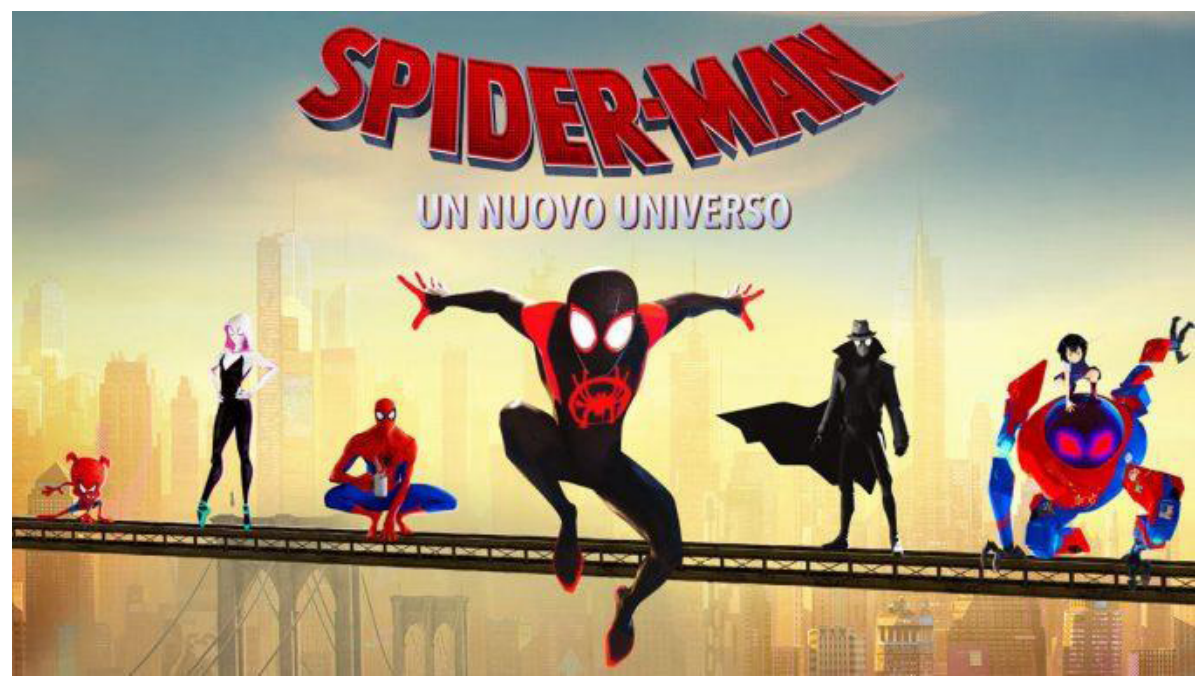

Figura 11. Immagine promozionale di Spider-Man: into the Spider-Verse con tutte le versioni coinvolte del personaggio.

Com'è visibile dalla figura 9, tutte queste versioni di Peter Parker possono rispondere a una stessa descrizione sommaria, ma nulla autorizza a indentificarli come la stessa persona; tuttavia, nel momento in cui indossano la maschera del loro alter-ego, diventano inconfondibili: il costume diventa quell'oggetto composto da semi nucleari plastici che rende l'Uomo Ragno un semema significante. Si può quindi dire che "Peter Parker" è l'insieme di semi nucleari fisici che vanno (molto spesso) a porsi all'interno del lessema "Uomo Ragno".

La figura 10 mostra come questo si riverberi anche sul grande schermo, dove i volti degli interpreti di Peter Parker differiscono fortemente, pur mantenendosi sempre simile il costume. Portando questo all'estremo, l'Uomo Ragno resta tale a prescindere da chi ne indossi la maschera, come visto recentemente nel film Spider-Man: into the Spider-Verse (Spider-Man. Un nuovo universo, Ramsey, Persichetti e Rothman, 2018).

Concludendo, quando la significazione di un personaggio è basata prevalentemente su semi nucleari plastici, non importa quali volti vi siano sotto: potranno quindi esistere Capitan America di ogni etnia, Thor uomo e donna, ecc. e, allo stesso tempo, sarà possibile efficacemente dar loro vita sul grande schermo.

\section{Ai confini del personaggio}

\subsection{Si vive solo due volte?}

Nato nel 1953 dalla fantasia di Ian Fleming e consacrato a icona della spy story dalla serie di film prodotti dalla EON, James Bond, alias Agente 007, presenta una notevole quantità di problemi a livello identitario. In quanto personaggio letterario, Bond nasce con caratteristiche fisiche poco definite: 


\section{Ocula $^{22}$}

Vol 21, No 22 (April 2020) • DOI: 10.12977/ocula2020-17

Nicolò Villani | Indagine identitaria del personaggio seriale. Verso una semiotica identitaria nella serialità

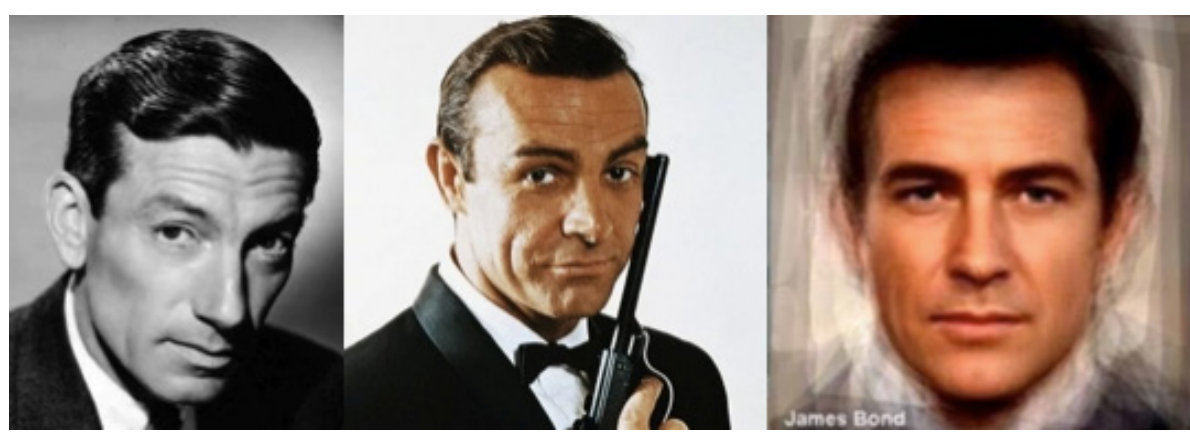

Figura 12. Da sinistra, Hoagy Carmichael, Sean Connery e un volto ricavato dall'insieme dei volti di tutti gli attori che hanno interpretato Bond.

nel primo romanzo della collana, Fleming ([1953] 2012: 47, tr. it.) raramente descrive Bond, limitandosi a poche frasi, sottolineando la somiglianza con Hoagy Carmichael e descrivendone brevemente occhi, capelli e una cicatrice sul viso (ivi: 64). Con il primo adattamento EON della serie sul grande schermo, Dr. No (Agente 0o7 - Licenza di uccidere, Young, 1962), ${ }^{8}$ ''agente segreto viene interpretato da Sean Connery, che sembra vestirne perfettamente i panni.

Connery interpreterà, però, solo sei pellicole tra le venti che compongono la serie cinematografica classica del personaggio; ${ }^{9}$ qui si apre il problema: James Bond sembra potersi permettere di fare a meno dell'imprescindibilità dell'effetto sosia descritto nel paragrafo 3.3 attivato dal medium cinematografico. Una possibile motivazione si può cercare con il primo cambio di attore: George Lazenby pronuncia infatti la celebre frase «Non era mai successo a quello di prima», alludendo al rifiuto di una ragazza da lui appena salvata; chi è "quello di prima" di cui si parla? Si può azzardare l'ipotesi che non solo "007" sia un nome in codice, ma che persino l'identità di "James Bond" sia un alias adottato da agenti diversi. L'ipotesi, in accordo con quanto detto nel paragrafo precedente, sembrerebbe l'unica in grado di spiegare il cambio di volto. Questa viene comunque smentita: la pellicola con Lazenby protagonista segna un momento cruciale nella storia di Bond, la morte della moglie appena sposata, evento sottolineato in film successivi, dopo un ulteriore cambio di volto (quello di Roger Moore). Sta quindi solo nella sua funzione all'interno del racconto (cfr. Eco [1976] 2013: 145 sgg.) l'identità di Bond? Se così fosse non si potrebbe pensare a una Bond-icona autonoma.

Esiste, però, tutto un insieme di elementi che stanno a metà tra il personaggio e il contesto in cui è inserito e che nel caso di Bond ricoprono un ruolo cruciale per la sua fortuna; questi sono gli «oggetti (automobili, armi, vestiti), elencati [...] con perizia enciclopedica da centinaia di siti e pubblicazioni [...] fino all'elemento più caratterizzante e citato di tutti, il James Bond Theme» (Pollone 2016: 11 sgg.).

8 La prima effettiva apparizione del personaggio in un audiovisivo risale al 1954, con Casino Royale, episodio della serie antologica Climax! (CBS, 1954-1958).

9 Non si considera qui il rilancio del personaggio avvenuto con Casino Royale (Id., Campbell, 2006) che sembra azzerare quanto raccontato nei film precedenti. 


\section{Ocula $^{22}$}

Vol 21, No 22 (April 2020) • DOI: 10.12977/ocula2020-17

Nicolò Villani | Indagine identitaria del personaggio seriale. Verso una semiotica identitaria nella serialità

Prima di analizzare lo specifico di questi elementi e come agiscono sull'identità del personaggio, occorre osservare un ulteriore universo narrativo in cui il cambio di volto è parte integrante della narrazione: Doctor Who.

5.2. «Dottore... Chi?»

Se in venti pellicole 007 è stato impersonato da cinque attori diversi, il Dottore, protagonista del franchise britannico Doctor Who, ha "indossato" in oltre cinquantacinque anni di vita almeno quattordici volti differenti tra loro, con la particolarità di fare di questo cambio un elemento strettamente diegetico: il Dottore ha, infatti, il potere di rigenerare sé stesso in punto di morte. ${ }^{10}$

In relazione a quanto precedentemente detto, vi sono diverse conseguenze: ogni incarnazione subisce un fortissimo effetto sosia rispetto all'attore che lo interpreta, tanto che ciascuno dei volti del Signore del Tempo viene nominato con il proprio numero identificativo, mantenendo il relativo aspetto in ogni trasposizione transmediale; ogni incarnazione ha, inoltre, una spiccata differenziazione plastica che la renda facilmente distinguibile: ciascun Dottore indossa uno o più outfit caratteristici che ne avvolgono la significazione; dal punto di vista caratteriale, ogni interprete personalizza l'agire del proprio Dottore.
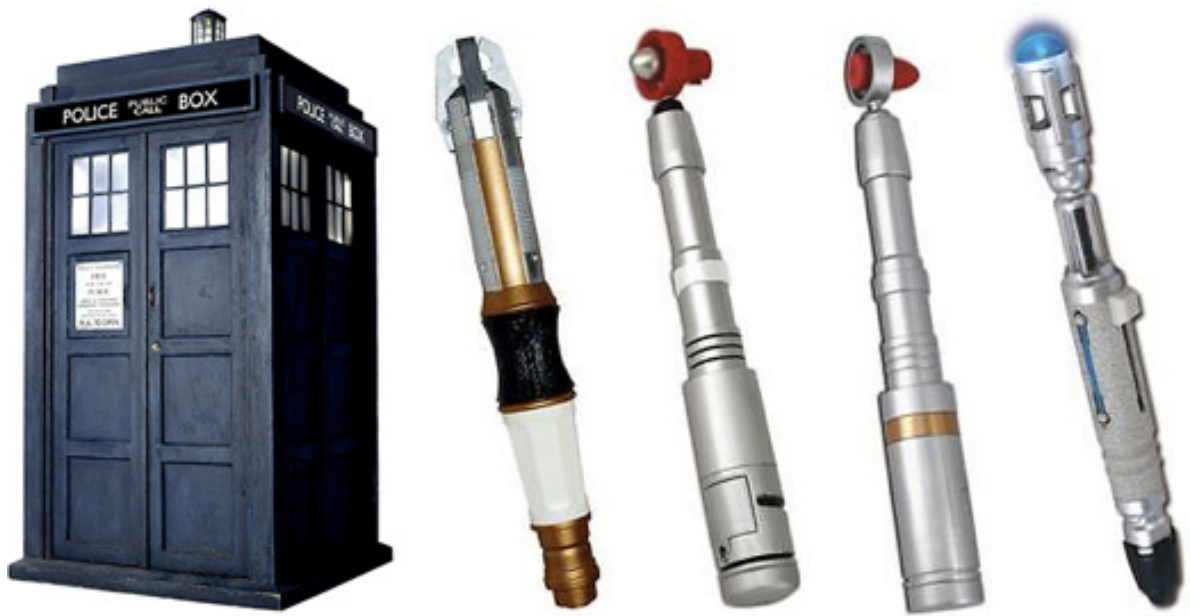

Figura 13. Da sinistra, il TARDIS e alcuni modelli di cacciavite sonico.

A tutto questo si aggiunge il fatto che il Dottore non esplicita mai il proprio nome (cfr. Hills 2010: 55). Dove va cercata, quindi, la «Doctor Who-ness» (ivi: 32) che consolida quella "textual "essence"» (ivi: 33) che rende il Dottore univocamente significante? Applicando l’ipotesi "oggettuale" suggerita

10 Questa strategia è stata introdotta necessariamente dal momento che William Hartnell era troppo anziano per portare avanti il personaggio all'inizio della quarta stagione della Serie Classica. 


\section{Ocula}

Vol 21, No 22 (April 2020) • DOI: 10.12977/ocula2020-17

Nicolò Villani | Indagine identitaria del personaggio seriale. Verso una semiotica identitaria nella serialità

per Bond, si può verificare come anche in Doctor Who esista un insieme di elementi "a metà" tra personaggio e contesto: dai temi musicali, agli oggetti indelebilmente legati alla serie, come il TARDIS o il cacciavite sonico, con cui spesso il Signore del Tempo riconosce sé stesso in altre incarnazioni. ${ }^{11}$

Tutti questi elementi sono classificabili semi nucleari circostanti, i quali hanno un peso considerevole nell'identificare il personaggio.

\subsection{Evanescenza e circostanza}

Immaginando il contesto come una "bolla" che circonda l'ipotesi puntiforme del personaggio, resta un'area vuota all'interno di essa: questa comprende gli elementi che stanno a metà tra personaggio e contesto, che sembrano potersi "staccare" dal primo ed essere proiettati verso l'esterno, pur mantenendo un forte rimando di significazione; questi elementi sono i semi nucleari circostanti, chiamati così perché creano un "intorno" del personaggio attraverso cui si irradia la significazione.

Si sono fin qui individuate tipologie di semi nucleari circostanti fortemente eterogenei, che danno, però, origine a un medesimo effetto di "evanescenza" del personaggio.

Si hanno le frasi ricorrenti, che aiutano a confermare l'identità del personaggio nel momento in cui vengono pronunciate: nel caso di 007, «il mio nome è Bond... James Bond», nel caso del Dottore, «io sono il Dottore» (frase che riceve spesso in risposta la domanda «Dottore... Chi?»). Comprimari e oggetti ricorrenti sembrano avere una funzione simile: tanto i colleghi di James Bond (M. e Moneypenny in primis), quanto i nemici classici del Dottore (cfr. Hills 2006: 116-140) riconoscono il loro rispettivo personaggio di riferimento, autorizzando lo spettatore a fare lo stesso. Allo stesso modo, gli oggetti ricorrenti portano con loro rimandi inscindibili col personaggio: così una Walther PPK resterà la pistola di 007 anche se usata in un'altra serie $\mathrm{e}^{12} \mathrm{e}$ una cabina blu andrà a indicare la presenza del Dottore vicino a dov'è parcheggiata. In ultimo la musica, che può spesso richiamare in scena un personaggio, sia questi presente o meno (cfr. Pollone 2016: 18).

Osservando brevemente l'azione di queste tipologie di semi nucleari, appare evidente una loro caratteristica unica: essi sono in grado di farsi carico autonomamente della significazione del personaggio e diventarne veicoli che si distaccano da esso (ibidem). I semi nucleari appaiono quindi come sorte di "veicoli di débrayage", al momento in cui sono caricati del significato del personaggio, diventandone così deittici all'interno di qualsiasi testo, senza la necessità che il personaggio sia presente insieme a loro. Attraverso i semi nucleari circostanti il personaggio si trova a vivere una natura "evanescente", in quanto la sua presenza è percepibile anche se non tangibile.

11 Si vedano come esempi gli episodi School Reunion (stagione 2, episodio 3, Serie Nuova) e The Day of the Doctor (stagione 7, episodio 14, Serie Nuova).

12 Il riferimento è al terzo episodio della seconda stagione di Twin Peaks (I Segreti di Twin Peaks, ABC, 1990-2017). 


\section{Ocula ${ }^{22}$}

Vol 21, No 22 (April 2020) • DOI: 10.12977/ocula2020-17

Nicolò Villani | Indagine identitaria del personaggio seriale. Verso una semiotica identitaria nella serialità

\section{Conclusioni}

Da quanto si è detto fin ora si può stilare una, seppur approssimativa e non vincolante, classificazione delle forme espressive esaminate e dei semi nucleari che maggiormente concorrono in esse ad affermare l'identità del personaggio. Per quanto riguarda il medium letterario, la significazione necessita di essere legata ai semi nucleari caratterizzanti, che determinano il comportamento e le relazioni del personaggio; ogni altra componente semica può essere presente, per quanto sempre vittima di quell'imprescindibile indefinitezza delle descrizioni verbali. Il medium letterario è anche efficace per quelle tipologie di semi nucleari circostanti come frasi tipiche e personaggi comprimari. Il medium fumettistico soffre dell'approsimatività dei disegni e delle differenze che possono intercorrere tra gli stili di più disegnatori; l'unica scappatoia è l'utilizzo dei semi nucleari plastici per affermare l'identità dei personaggi; i fumetti, essendo un linguaggio "a metà" tra il letterario e l'audiovisivo (Barbieri 1991) danno spazio tanto ai semi nucleari efficaci in letteratura, quanto a quei tipi di semi nucleari circostanti rappresentanti oggetti ricorrenti e ben riconoscibili, che si incaricano di veicolare l'identità del personaggio. In ultimo, il medium audiovisivo, in tutte le sue possibili declinazioni, oltre a comprendere tutti i semi nucleari sopra elencati, permette anche l'attivazione dell'effetto sosia veicolato dai semi nucleari fisici; in aggiunta, vengono compresi quei semi nucleari circostanti concernenti la musica, sia questa diegetica o meno. A questa sorta di "sintesi additiva" dei semi nucleari va poi compreso il pressoché imprescindibile utilizzo dei semi contestuali, che entrano in gioco al momento della narrazione; l'obiettivo di questa classificazione è comprendere dove individuare l'identità del personaggio a prescindere dal contesto e dalle strutture narrative in cui va inserito, pur consapevoli che il personaggio seriale è prima di tutto un contenuto da veicolare; si conferma pertanto essere il medium a dettare le regole per rendere il personaggio un semema pieno, significante e riconoscibile.

\section{Bibliografia}

AA.VV.

2008 Marvel Chronicle, Modena, Panini Comics.

Bandilari, Luca; Terrone, Enrico

2009 Il sistema sceneggiatura, Torino, Lindau.

Barbieri, Daniele

1991 I linguaggi del fumetto, Milano, Bompiani.

Barthes, Roland

$1970 S / Z$, Paris, Seuil (tr. it. S/Z. Una lettura di «Sarrasine» di Balzac, Torino, Einaudi, 1973).

Bertieri, Claudio

1979 I film di carta, Firenze, Vallecchi. 


\section{Ocula ${ }^{22}$}

Vol 21, No 22 (April 2020) • DOI: 10.12977/ocula2020-17

Nicolò Villani | Indagine identitaria del personaggio seriale. Verso una semiotica identitaria nella serialità

Bisoni, Claudio

2018 "Pubblici, utenti, spettatori: il ruolo dell'audience nell'ecosistema dei media", in Pescatore G. (a cura di), Ecosistemi narrativi, Roma, Carrocci.

Bonvecchio, Claudio

2011 La filosofia di Indiana Jones, Milano-Udine, Mimesis.

Casetti, Francesco; Di Chio, Federico

1990 Analisi del film, Milano, Bompiani, 2009 ${ }^{16}$.

Codeluppi, Vanni

2015 Eroi. Superman, Batman, Tex, oo7, Harry Potter e altre figure dell'immaginario, Milano, Franco Angeli.

Comand, Maria Pia

2012 I personaggi dei film, Padova, Marsilio.

Cucchetti, Alice

2014 "Keep Flying. Appunti sui fenomeni di fandom", in Martin, S. (a cura di), La costruzione dell'immaginario seriale contemporaneo, Milano-Udine, Mimesis.

Doyle, Arthur Conan

1887 A Study in Scarlet, London, Ward Lock \& Co (tr. it. Uno studio in rosso, Milano, Mondadori, 2010).

Dusi, Nicola

2003 Il cinema come traduzione, Torino, Utet, 2019.

2015 Contromisure. Trasposizioni e intermedialità, Milano-Udine, Mimesis.

Eco, Umberto

1964 Apocalittici e integrati, Milano, Bompiani, 2013.

1976 Il superuomo di massa, Milano, Bompiani, 2005.

Fleming, Ian

1953 Casino Royale, London, Johnatan Cape (tr. it. Casino Royale, Milano, Adelphi, 2012).

Greimas, Algirdas Julien

1983 Du sens II, Paris, Seuil (tr. it. Del senso 2, 1998).

1984 "Sémiotique figurative et sémiotique plastique”, Actes Sémiotiques.

Documents, 60, Paris (tr. it. "Semiotica figurativa e semiotica plastica”, tr. it. in Corrain, Lucia e Valenti, Paolo, a cura di, Leggere l'opera d'arte. Dal figurativo all'astratto, Bologna, Esculapio, 2002, pp. 33-51).

Hills, Matt

2010 Thriumph of a Time Lord. Regenerating Doctor Who in the twenty-first century, London, I.B. Tauris.

Hjelmslev, Louis

1961 Prolegomena to a Theory of Language, Madison, University of Wisconsin Press (tr. it. I fondamenti della teoria del linguaggio, Torino, Einaudi, 1968).

Innocenti, Veronica; Pescatore, Guglielmo

2008 Le nuove forme della serialità televisiva. Storia, linguaggio e temi, Bologna, Archetipolibri. 


\section{Ocula ${ }^{22}$}

Vol 21, No 22 (April 2020) • DOI: 10.12977/ocula2020-17

Nicolò Villani | Indagine identitaria del personaggio seriale. Verso una semiotica identitaria nella serialità

Jenkins, Henry

2006 Convergence Culture, New York, NYU Press (tr. it. Cultura convergente, 2014).

2010 "La vendetta dell'unicorno origami”, Link, 9, Milano.

Marrone, Gianfranco

2003 Montalbano. Affermazioni e trasformazioni di un eroe mediatico, Torino, Rai-Eri.

Marsciani, Francesco

2012 Minima semiotica, Milano-Udine, Mimesis.

Marsciani, Francesco; Zinna, Alessandro

1991 Elementi di semiotica generativa, Bologna, Esculapio.

Pearson, Roberta

2016 "Googling Sherlock Holmes", in Pesce, S. e Noto, P. (a cura di), The Politics of Ephemeral Digital Media: permanence and obsolescence in paratext, Abingdon-on-Thames, Routledge.

Pollone, Matteo

2016 "La 'formula Bond'. Un'introduzione e una visione d'insieme", in Pollone, M. (a cura di), James Bond. Fenomenologia di un mito (post)moderno, Milano, Bietti.

Pozzato, Maria Pia

2014 Capire la semiotica, Roma, Carrocci.

Semprini, Valentina

2006 Bam! Sock! Lo scontro a fumetti, Latina, Tuné.

Tralli, Lucia

2018 "Pratiche delle audience nell'ecosistema narrativo", in Pescatore G. (a cura di), Ecosistemi narrativi, Roma, Carrocci.

Nicolò Villani è laureato triennale al DAMS di Bologna con una tesi in Semiotica dei Media e magistrale al CITEM in Storia della Serialità. Tra i suoi interessi spiccano la Semiotica generativa, il panorama seriale contemporaneo, lo sviluppo dei media digitali e la possibilità di riportare l'indagine strutturale (in particolar modo con gli sviluppi più recenti dell'Etnosemiotica) a incontrare lo studio della testualità audiovisiva contemporanea. Reduce da un'esperienza come giudice alla 75a Mostra del Cinema di Venezia per la sezione Venezia Classici, è redattore nella rivista di cinema e televisione Birdmen. 